\title{
Management Outcome of Infancy and Childhood Intussusception in Rajshahi Medical College Hospital - A Prospective Study
}

\author{
M A Nowshad ${ }^{1}$, A Moshtaque ${ }^{2}$, R Hafizur $^{3}$
}

\begin{abstract}
Background: Intussusception is one of the major causes of bowel obstruction in infancy and childhood. Proper diagnosis and effective management have significantly reduced its mortality and morbidity in developed country. Aim: To document the mode of presentation and treatment outcome of Intussusception at Rajshahi Medical College Hospital. Patients and Methods: Thirty three consecutive cases of this condition seen at paediatric surgical ward over one year period were prospectively studied. Details of symptoms and signs, pre-hospital care, treatment and outcome were documented. Result: The triad of abdominal pain, bloody mucoid stools and palpable abdominal mass was seen in $24(72 \%)$ of the cases. Twenty one (63\%) had been admitted and treated with intravenous fluids in peripheral health centre for an average of 3 days before referral to this hospital. Prolonged mean duration of recognizable symptoms of 3 days accounted for a 58\% bowel resection rate. Wound infection occurred in 11(33.3\%) and burst abdomen in $07(21.2 \%)$ of cases. Faecal fistulae developed in $02(06 \%)$ and incisional hernia developed in $03(09 \%)$ of the patients. Overall, mortality rate was $09 \%$. Conclusion: the early symptoms of Intussusceptions would seem to be missed by primary health workers, with consequently high morbidity and mortality. There is an urgent need to re- emphasize these symptoms to the first line healthcare providers and parents through public enlightenment campaigns.
\end{abstract}

TAJ 2009; 22(1): 59-63

\section{Introduction}

Intussusceptions, a pathological telescoping of a portion of bowel into an adjacent part, is one of the commonest emergencies in infancy and childhood ${ }^{1}$. The etiology may be idiopathic or secondary to some pathology within the wall of the bowel. The primary (idiopathic) type, mostly seen in infancy and childhood, is ascribed to nonspecific viral origins, whereas in older children and adults Intussusceptions is usually secondary to some identifiable intramural or itraluminal pathologies such as polyps, lipomas and malignancies ${ }^{2,3}$. Children may present at any age but this occurs most commonly in the first year. The mode of presentation may vary depending upon the time of presentation. Late presentation as a cause of unacceptably high morbidity and mortality rate in Intussusceptions has been documented from many African centers ${ }^{4,5,6,7}$. Many past studies on this condition from different region were retrospective and we therefore embarked on this prospective study to document the presentation and management outcome of this condition in Rajshahi Medical College Hospital.

${ }^{1}$ Assistant Professor, Department of Pediatric Surgery, Rajshahi Medical College, Rajshahi.

2 Professor, Department of Pediatric Surgery, Rajshahi Medical College, Rajshahi.

${ }^{3}$ Assistant Professor, Department of Radiology \& Imaging, Rajshahi Medical College, Rajshahi. 


\section{Material and method}

This study was performed in paediatric surgery department of Rajshahi Medical College Hospital, from July 2008 to June 2009. We prospectively kept record of all children presenting with Intussusceptions, detailing the children's presenting symptoms and signs, symptoms duration as well as initial care obtained from the referring hospitals in a protocol. The clinical profile of each patient on admission was noted while mode of treatment and outcome of management were documented. For those who proceeded to surgery, operative findings, procedure performed, peri-operative complications, duration of hospitalization and prognosis were all recorded. Data were analyzed on SPSS 9.0. Mean and standard deviation were computed with the student's t-test, whereas nonparametric variables were analyzed by Chi-square method and unless otherwise stated, $\mathrm{p}<0.05$ was taken as significant.

\section{Results}

Total 33 children were admitted in one year period. There were 21 (64\%) male and 12(36\%) female, with a male to female ratio of 1.7:1. Mean weight was $9.3 \pm 2.3 \mathrm{Kg}$. The age ranged from 3 month to 5 years.

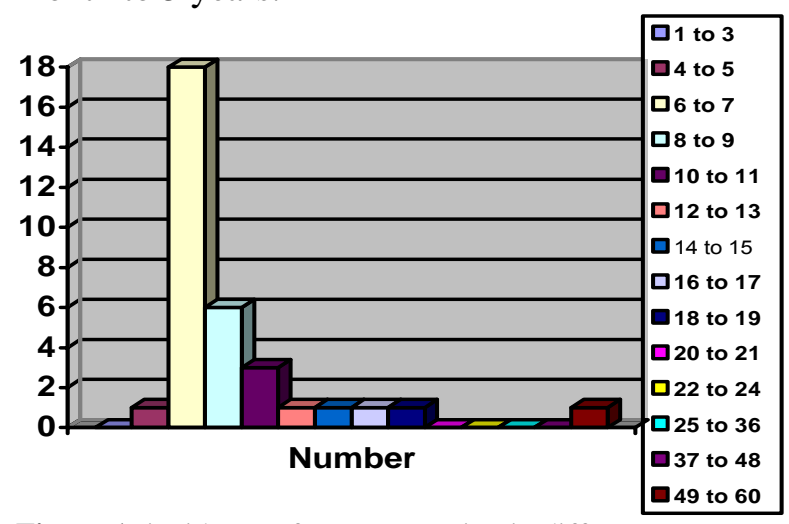

Figure 1: incidence of Intussusception in different age group,

The modal age was 6 months, during which 18 (54.5\%) of all cases occurred. The peak incidence was between 6 and 7 months. Eight (24.2\%) of the patients were still breast feeding, eighteen (54.5\%) were weaning, whereas $7(21.2 \%)$ had been weaned on presentation. Peak seasonal presentation occurred in July and September coinciding with the period where the rain / cold season interchange in Bangladesh.

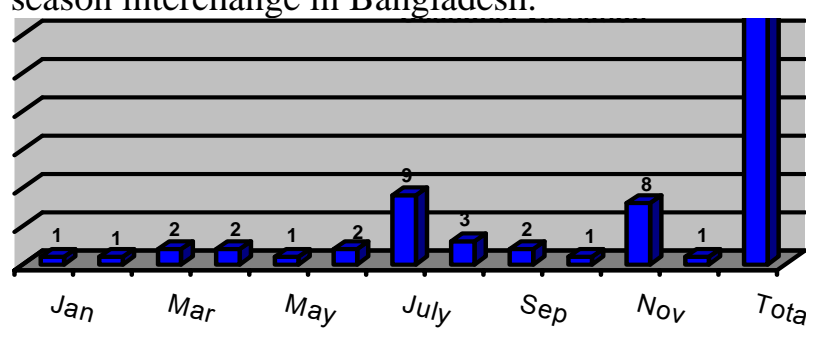

Figure 2: Peak seasonal presentation of Intussusception.

Abdominal pain, vomiting and bloody stool had occurred for duration of 3-9 days, with a mean duration of $4.8+1.8$ days. The classical triad of abdominal pain, bloody mucoid stools and a palpable abdominal mass was seen in 22 (66.6\%) of cases. Upper respiratory tract infection occurred in $8(24.2 \%)$ patients. In patients with nonpalpable abdominal masses there was gross abdominal distension and guarding. In 15 (45.4\%) patients, the abdominal masses were palpable per rectum. one (03\%) patients presented with prolapsing masses at the anus. These were significantly related to delayed presentation of more than 5 days $(\mathrm{p}<0.01)$.

Twenty eight (84.8\%) patients had been previously admitted in to paediatric medicine unit or private hospital prior to presentation at paediatric surgery ward. Nineteen (57.6\%) of them treated by the village doctor initially for 2-3 days and had been on antibiotics. Five (15.1\%) patients had received no treatment prior to hospitalization. Eleven (33.3\%) presented in shock were septic, oliguric or moribund.

Intravenous fluid resuscitation, correction of electrolyte derangement, antibiotic therapy and early surgical exploration were the main thrust of our management. Plain abdominal X-ray was obtained in all patients. Abdominal ultrasonography (USG) was performed in 22 (66.6\%) of patients, rest was seldom indicated because of delayed presentation. Surgical intervention time ranged from 96 to 180 hours, with a mean of $156 \pm 18$ hours. All patients obtained treatment within 48 hours of admission. 
Infrastructural delays, financial difficulties and prolonged resuscitation needs in moribund patients were responsible for delayed intervention in this series. Hydrostatic reduction was not attempted in our patients.

Surgical exploration was the mainstay of treatment. Ileocolic intussusceptions was seen in $27(81.8 \%)$ of all cases that had laparotomy, the colocolic variety was recorded in 5 (15.1\%) cases, whereas ileo-colocolic type was seen in one (03\%).

In 22 (66.6\%) patients, manual reduction was attempted at surgery and 8 (36\%) of these attempts were successful. In one patient (03\%) intussusceptions spontaneously reduced at laparotomy. Total 25 (75.7\%), 14 unsuccessful reduction and another 11 deemed outright unfit for manual reduction at laparotomy, underwent bowel resection and end to end anastomosis.

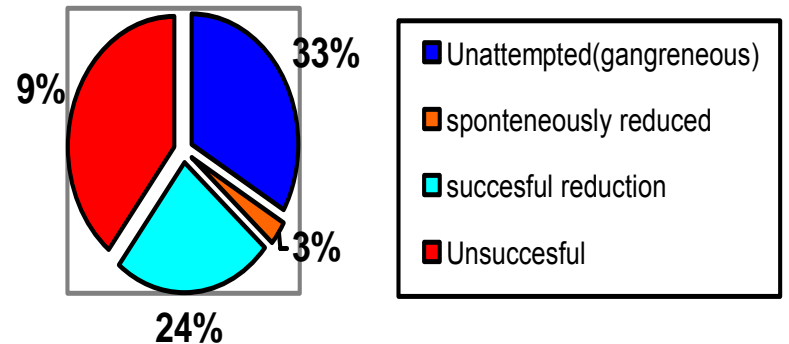

Figure 3: Operative management of Intussusception.

Eighteen (54.5\%) of these patients had right hemicolectomy whereas in 7 (21\%) others, limited ileal resection was performed.

Out of 33 cases recorded, 12(36.3\%) resulted from non-specific lymphadenitis. Three (9\%) cases were seen solitary polyp in the resected part that probably acts as lead point.

Mean duration of hospitalization period was 15+ 9.6 days with a range of 9 days to 21 days. Wound sepsis was the commonest complication, occurring in $9(27.2 \%)$ of all cases. Burst abdomen occurred in $5(15.1 \%)$ whereas fecal fistulae was seen in $1(03 \%)$ of our cases. There was no recurrence of intussusceptions in this series. Late complications seen in our patients were stitch granuloma 3(9\%), incisional hernia 2(6\%) and post operative adhesive bowel obstruction in 2(6\%) cases. In our study follow-up period was 6 month to 2 years.

Total mortality was 3 in this series with a mortality rate $9 \%$. Two (6\%) of these had died in the post-operative period from overwhelming sepsis and another 1 (03\%) due to anesthetic complication. Mean duration of abdominal pain, vomiting and bloody mucoid stools were significantly greater for patients who died compared with that of the survivors.

Table I: Comparison of duration of symptoms and outcome of Intussusceptions.

\begin{tabular}{llllll|}
\hline Symptom & \multicolumn{2}{l}{ Survivors(n=30) } & Dead $(\mathrm{n}=3)$ & P value \\
\cline { 2 - 4 } & $\begin{array}{l}\text { Mean duration } \\
\text { (Days) }(\mathrm{SD})\end{array}$ & $\begin{array}{l}\text { Mean } \\
\text { duration } \\
\text { (Days) } \\
\text { (SD) }\end{array}$ & \\
\hline $\begin{array}{l}\text { Abdominal } \\
\text { pain }\end{array}$ & 4.8 & 2.7 & 6.2 & 1.8 & $<.01$ \\
Vomiting & 4.1 & 2.2 & 6.8 & 2.1 & $<.01$ \\
Bloody stool & 2.6 & 2.1 & 4.2 & 1.8 & $<.001$ \\
\hline
\end{tabular}

\section{Discussion}

Rajshahi is a North Bengal city with about 1 million inhabitants. Rajshahi Medical College Hospital is a tertiary hospital having 35 beds for paediatric surgical patients. The patients are being referred to this hospital from surrounding 12 districts. From our record, we had observed that intussusceptions is one of the leading causes of intestinal obstruction in infants. The age range seen in our study was in keeping with universally quoted period, whereas the peak incidence at 6-9 months recorded matched what has been severally reported in local and international literatures ${ }^{8,9,10}$.

The seasonal variation we reported however portrays the peculiarity of primary intussusceptions as seen in many part of the world. The two peak periods of presentation coincide with the times of seasonal change in Bangladesh. July marks the onset of raining season while there is transition of dry and cold from November in each year. At each of this weather transition, there usually occurs an out break of viral upper respiratory tract infection and gastroenteritis providing an etiology for hypertrophy of Peyer's 
patches. There has been no definite association between the seasonal rates of rotavirus and intussusceptions $^{11,}{ }^{12}$. However one study of Nigeria, documented the presence of virus in $60.1 \%$ of affected children and isolated adenovirus in the stool of more than half of them, using monoclonal antibody ${ }^{13}$.

Abdominal colic, persistent vomiting and bloody mucoid stool occurred in over $66.6 \%$ of our cases. But unfortunately $84.8 \%$ of them were admitted in pediatric medicine unit or in private clinic and then referred in pediatric surgical unit after variable period of time. Many cases of this series however misdiagnosed as possible amoebic dysentery and sent back home on metronidazole and antibiotics. The delay occurred by this poor pre-referral cases led to a prolonged mean duration of 4.8 days. The attendant poor prognosis seen in $9 \%$ was largely as a result of this delay. Most authors from this sub-region have bemoaned the high mortality as a result of this delayed presentation of intussusceptions ${ }^{4,5,9}$. It is viewed from this study that, over $80 \%$ of our patients had presented early in primary healthcare points, but were misdiagnosed and wrongly given ambulatory therapy for suspected infected gastroenteritis. This therefore calls for an urgent awareness campaign among doctors, nurse and parents to raise the index of suspicion of and increase the rate of early detection of this condition. In a well-designed comparative study of management outcome of intussusceptions between a rural African setup and an American metropolis hospital, attributed the $18 \%$ mortality in the Africans due to delayed presentation and consequent gangrenous bowel and opined that non-operative reduction would have achieved little in this group of moribund late presenters ${ }^{14}$.

Our management priorities comprising fluid resuscitation, correction of electrolyte derangements, antibiotic therapy and early surgical exploration were sometimes constrained by institutional, financial and patient related factors. As a typical of many, a developing country set-up, $\mathrm{RMCH}$ is not free of power outages, shortage of consumables and other institutional bottlenecks traceable to the absence of committed, workable governmental policies on healthcare funding. Our experience in this regard was similarly too many reports from sub-Saharan Africa. Adejuyigbe et at ${ }^{16}$ attributed the high rate of bowel resection and mortality in intussusceptions managed in Nigeria due to delayed presentation. Similar conclusions have made by others who unanimously advocated improved awareness of intussusceptions among stakeholder to reduce its unacceptably high mortality in Africa ${ }^{17,18,19,20 .}$

Abdominal ultrasonography was done when it was available. Barium enema, which could be used to diagnose and pressure reduce the intussusceptions, was not performed in these very ill children because it is contraindicated in prolonged delay where gangrenous bowel could easily perforate. It is hoped that with improved awareness of this condition and consequent increase in the number of early presentations, future studies will elucidate the benefit of this treatment modality on selected cases in our centre.

The complications recorded in our study were typical of our third world setting. Wound infection was really a bother in delayed arrival cases. Wound dehiscence cases needed secondary closer after control of sepsis.

Mortality rate of $9 \%$ needs to be improved upon through prompt diagnosis and early referral. This figure is however comparable to similar rates obtained from other workers in different part of the world, where rates of between 8 and 25\% have been recorded ${ }^{6,141618,21}$. Harouna et al ${ }^{5}$ observed a heavy $55 \%$ mortality among cases of pediatric intussusceptions in Niamey, Republic of Niger. This was attributed to delayed presentation and advanced peritonitis, coupled with inadequate facilities to manage these challenging cases.

It is concluded from this study that delayed referral from the primary care provider is a major cause of prolonged duration of symptoms and the attendant high morbidity and mortality in childhood intussusceptions seen in this region. This delay stems from misdiagnosis of the condition and effort should be made to be improving awareness at both parental and primary care level to promote early presentation and referral. 


\section{Reference}

1. Sanjay NO \& Nitin GC. Intussusception. Paediatric Surgical Diagnosis. Jaypee Brothers Medical Publisher (p) Ltd.

2. Baracchini A, Chiaravalloti G, Quiniti S, Favili T, Ughi C. Intestinal intussusception in children . Minerva Pediatr 1995; 47: 215-219.

3. Courtney SP, Ibrahim N, Longstuff AF \& Davidson CM. Intussusception in the adult: Clinical, Radiological and Histological features. Postgrad Med J 1992; 68: 449- 452

4. Grant HW, Buccimazza I, Hadly GP. A comprison of colo-colic and ileo-colic intussusception. J Pediatr surg 1996; 31: 1607-1610.

5. Harouna $Y$, Tardivel G, abdou I, Gamatic Y, Mariama $S$ \& Bia $M$. prognosis of acute intestinal intussusception in infant at the national hospital of Niamey (Niger): Eleven cases treated surgically. Bull Soc Pathol Exot 1997; 90: 30-32.

6. Mangete ED \& Allison AB. Intussusception in infancy and childhood: An analysis of 69 cases, West Afr J med 1994; 13: 87-90.

7. Gudeta B. Intussusception in children: A ten year reiew. East afr Med 1993; 70: 730-731.

8. Crankson SJ, Al-Rabeeah AA, Fischer JD, AlJadan SA \& Namshan MA. Idiopathic intussusception in infancy and childhood. Soudi Med J 2003; 24: 18-20.

9. Ameh EA. The morbidity and mortality of right hemicolectomy for complicated intussuscepton in iffants. Niger Postgrad Med J 2000; 9: 123-124.

10. Adebamowo CA, Akang EE, Pindiga HU, Ezeome ER, Omotosho Po, Labeodan OA et al. Chanhing clinicopathological profile of intussusception in Nigeria: A 20 year review. Hepatogastroenterology 2000; 47: 437-440.

11. Nelson EA, Tam JS, Glass RI, Parashar UD, Fok TF. Incidence of rotavirus diarrhea and intussusception in Hong Kong using standardized hospital discharge data. Pediatr Infect Dis J 2002;21:701-3

12. Perez-Schael I, Escalona M, Salinas B, Materan M, Perez ME, Gonzalez G. Intussusception-associated hospitalization among Venezuelan infants during 1998 through 2001: Anticipating rotavirus vaccines. Pediatr Infect Dis J 2003; 22:234-9.

13. Bode CO, Omilabu SA. Viral isolates of intussusception in Nigerian infants. S Afr J Surg 2002; 40:57-8.

14. Meier DE, Coln CD, Rescorla FJ, OlaOlorun A, Tarpley JL. Intussusception in children: International perspective. World J Surg 1996; 20:1035-9.

15. Rahman GA, Mungadi IA. Gangrenous bowel in Nigerians. Cent Afr J Med 2000;46:321-4.

16. Adejuyigbe $O$, Jeje EA, Owa JA. Childhood intussusception in lle-Ife, Nigeria. Ann Trop Paediatr 1991; 11:123-7.

17. Isdale JM, Saunders WC. Intussusception in Johannesburg: A review of 81 cases. S Afr Med J 1986; 69:610-1.

18. Odita JC, Piserchia NE, Diakporomre MA. Childhood Intussusception in Benin City, Nigeria. Trop Geogr Med 1981; 33:317-21.

19. Edino ST, Ochicha O, Mohammed AZ, Anumah M. Intussusception in Kano: A 5-year analysis of pattern, morbidity and mortality. Niger $\mathrm{J}$ Med 2003;12:221-4

20. Daniel E, Melaku G, Yoo MC, Agzew Y, Gebre W. Analysis of surgical admissions to the EthioSwedish Children's Hospital (1984-1988) in Addis Ababa. Ethiop Med J 1990; 28:15-22.

21. Ameh EA. The morbidity and mortality of laparotomy for uncomplicated Intussusception in children. West Afr J Med 2002; 21:115-6. 